\title{
Benefits and Risks of Clopidogrel vs. Aspirin Monotherapy after Recent Ischemic Stroke: A Systematic Review and Meta-Analysis
}

\author{
Maurizio Paciaroni $\mathbb{D}^{1}{ }^{1}$ Birsen Ince, ${ }^{2}$ Bo Hu, ${ }^{3}$ Jiann-Shing Jeng, ${ }^{4}$ Kursad Kutluk, ${ }^{5}$ \\ Liping Liu $\mathbb{D}^{6},{ }^{6}$ Min Lou, ${ }^{7}$ Vladimir Parfenov, ${ }^{8}$ Ka Sing Lawrence Wong, ${ }^{9}$ Babak Zamani, ${ }^{10}$ \\ Dara Paek, ${ }^{11}$ Jung Min Han, ${ }^{11}$ Michael del Aguila, ${ }^{11}$ and Shalini Girotra ${ }^{12}$ \\ ${ }^{1}$ Stroke Unit and Division of Cardiovascular Medicine, University of Perugia, Santa Maria della Misericordia Hospital, Perugia, Italy \\ ${ }^{2}$ Department of Neurology, Division of Cerebrovascular Diseases, Cerrahpasa Medical Faculty, Istanbul University, Istanbul, Turkey \\ ${ }^{3}$ Union Hospital Affiliated to Tongji Medical College of Huazhong University of Science and Technology, Wuhan, China \\ ${ }^{4}$ Stroke Center and Department of Neurology, National Taiwan University Hospital, Taipei, Taiwan \\ ${ }^{5}$ Department of Neurology, Dokuz Eylul University, Izmir, Turkey \\ ${ }^{6}$ Department of Neurology and Stroke Center, Beijing Tiantan Hospital, Capital Medical University, Beijing, China \\ ${ }^{7}$ The Second Affiliated Hospital of Zhejiang University, School of Medicine, Hangzhou, China \\ ${ }^{8}$ Department of Nervous Diseases and Neurosurgery, Sechenov First Moscow State Medical University, Moscow, Russia \\ ${ }^{9}$ Department of Medicine and Therapeutics, The Chinese University of Hong Kong, Hong Kong \\ ${ }^{10}$ Iranian Stroke Society, Tehran, Iran \\ ${ }^{11}$ Doctor Evidence, Santa Monica, CA, USA \\ ${ }^{12}$ Sanofi, General Medicines and Emerging Markets, Singapore
}

Correspondence should be addressed to Maurizio Paciaroni; maurizio.paciaroni@unipg.it

Academic editor: Joel C. Marrs

Received 16 May 2019; Revised 16 August 2019; Accepted 17 September 2019; Published 1 December 2019

Copyright (c) 2019 Maurizio Paciaroni et al. This is an open access article distributed under the Creative Commons Attribution License, which permits unrestricted use, distribution, and reproduction in any medium, provided the original work is properly cited.

Aim. Though combination of clopidogrel added to aspirin has been compared to aspirin alone in patients with stroke or transient ischemic attack, limited data exists on the relative efficacy and safety between clopidogrel and aspirin monotherapy in patients with a recent ischemic stroke. We aimed to compare clopidogrel versus aspirin monotherapy in this population. Methods. PubMed, Embase, and CENTRAL databases were searched from inception to May 2018 to identify clinical trials and observational studies comparing clopidogrel versus aspirin for secondary prevention in patients with recent ischemic stroke within 12 months. Pooled effect estimates were calculated using a random effects model and were reported as risk ratios with $95 \%$ confidence intervals. Results. Five studies meeting eligibility criteria were included in the analysis. A total of 29,357 adult patients who had recent ischemic stroke received either clopidogrel $(n=14,293)$ or aspirin $(n=15,064)$ for secondary prevention. Pairwise meta-analysis showed a statistically significant risk reduction in the occurrence of major adverse cardiovascular and cerebrovascular events (risk ratio 0.72 [95\% CI, $0.53-0.97])$, any ischemic or hemorrhagic stroke $(0.76[0.58,0.99)$, and recurrent ischemic stroke $(0.72[0.55,0.94])$ in patients who received clopidogrel versus aspirin. The risk of bleeding was also lower for clopidogrel versus aspirin $(0.57[0.45,0.74])$. There was no difference in the rate of all-cause mortality between the two groups. Conclusions. The analysis showed lower risks of major adverse cardiovascular or cerebrovascular events, recurrent stroke, and bleeding events for clopidogrel monotherapy compared to aspirin. These findings support clinical benefit for single antiplatelet therapy with clopidogrel over aspirin for secondary prevention in patients with recent ischemic stroke.

\section{Introduction}

Stroke is the second most common cause of death and the third most common cause of disability worldwide [1]. Approximately 795,000 people in the United States experience a stroke each year, of which $87 \%$ are ischemic strokes [2]. Additionally, approximately $20 \%$ of patients with a primary diagnosis of stroke have a second stroke within two years, accounting for 185,000 annual cases in the United States [2,3]. Those with recurrent strokes have higher costs per patient and are more 
TABLE 1: Study inclusion and exclusion criteria in PICOTSS format.

\begin{tabular}{ll}
\hline Population & (i) Patients with recent ischemic stroke within the previous year \\
Interventions & (ii) Subgroup data for ischemic stroke patients in studies with mixed stroke/TIA populations \\
Comparators & Aspirin monotherapy (any dosage) for at least four weeks \\
& Efficacy outcomes \\
& (i) MACCE \\
& (a) Defined as any composite outcomes that included two or more of the following: recurrent stroke, myocardial infarc- \\
& tion, unstable angina, coronary revascularization, aortic aneurysm rupture, peripheral artery disease, vascular death and \\
& sudden death \\
& (ii) Recurrent stroke (ischemic and hemorrhagic) \\
& (iii) Recurrent ischemic stroke \\
Outcomes & (iv) Mortality \\
& Safety outcome \\
& (i) Bleeding risk \\
& (a) Intracranial \\
(b) Gastrointestinal \\
Timing & (c) Any reported \\
Setting & Minimum study duration/follow-up of at least four weeks (one month) \\
Study design & (i) Randomized controlled trials \\
& (ii) Comparative observational studies
\end{tabular}

MACCE: major adverse cardiovascular and cerebrovascular events; TIA: transient ischemic attack.

likely to experience poor outcomes as compared to patients with primary stroke $[2,4-6]$. Therefore, secondary stroke prevention in patients with a history of ischemic stroke is critical in reducing the overall burden of stroke. It is estimated that nearly $80 \%$ of secondary strokes can be prevented with antiplatelet therapy when combined with lifestyle changes [2, 7].

Current guidelines from the American Heart Association and American Stroke Association recommend antiplatelet therapy with aspirin for patients with ischemic stroke $[7,8]$. Other approved antiplatelet treatment options including clopidogrel, aspirin/dipyridamole, and ticlopidine have been shown to be safe and effective for secondary prevention in this population, however, the relative safety and effectiveness among the different antiplatelet agents has still not been clearly established [9-13]. Dual antiplatelet therapy with clopidogrel in combination with aspirin has been compared to aspirin in reducing recurrent stroke in patients with minor stroke (within 12-24 hours from onset) or transient ischemic attack (TIA) $[14,15]$, but data on the efficacy and safety of clopidogrel compared to aspirin as single antiplatelet agents exclusively in patients with recent ischemic stroke is limited. The aim of this study was to conduct a systematic review and meta-analysis to compare the efficacy and safety of clopidogrel versus aspirin used as a monotherapy for secondary prevention in patients with recent ischemic stroke. Findings from this comprehensive update on the available body of evidence will guide healthcare professionals and decision makers on the selection of optimal antiplatelet agent for preventative use in patients with recent ischemic stroke.

\section{Methods}

2.1. Data Sources and Searches. Literature searches were performed by a medical librarian (HT) in PubMed, Embase, and the Cochrane Central Register of Controlled Trials for studies published from inception to May 2018. Search strategies are provided in Supplemental Table I. Search results were exported to Digital Outcome Conversion $\left(\right.$ DOC $^{\mathrm{rm}}$ ) Library Management System (LMS, version 2.0), and duplicates were removed (Doctor Evidence, Santa Monica, CA) [16]. To ensure that potentially relevant studies were not overlooked, reference lists from other reviews and meta-analyses on the current topic were searched by hand. The Preferred Reporting Items for Systematic review and Meta-Analysis protocols (PRISMA) guidelines were followed [17].

2.2. Study Selection. Medical librarians screened titles and abstracts based on a standardized review protocol that defined study eligibility criteria using the PICOTSS format, which outlines the participants, interventions, comparators, outcomes, timing, setting, and study designs of interest (Table 1). Eligible studies were those that compared the beneficial and harmful effects of clopidogrel and aspirin monotherapies for the prevention of recurrent stroke and other cardiovascular complications in patients who experienced ischemic stroke in the previous year. Studies that also enrolled patients with TIA were included only if data for ischemic stroke patients were reported separately. Randomized controlled trials (RCT) and comparative observational studies with at least 1-month follow-up were included. There was no restriction for study setting. Studies were required to report at least one outcome of interest.

Efficacy outcomes included recurrent stroke of any type, recurrent ischemic stroke, and all-cause mortality. Also collected were major adverse cardiovascular and cerebrovascular events (MACCE). MACCE was defined as a composite outcome that included two or more of the following: recurrent stroke, myocardial infarction, unstable angina, coronary revascularization, aortic aneurysm rupture, peripheral artery 


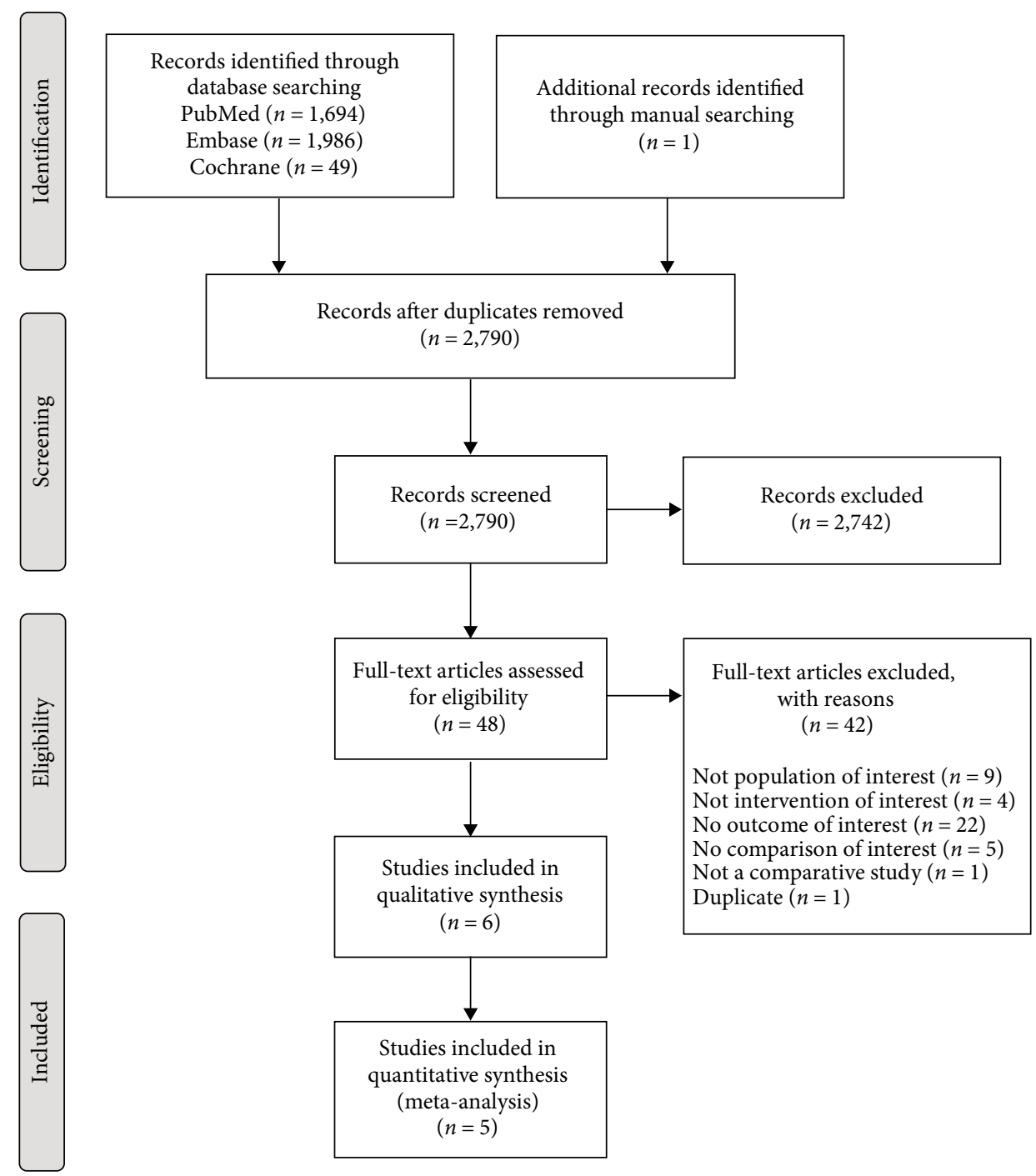

FIGURE 1: PRISMA flow diagram showing study identification and selection.

disease, vascular death, and sudden death. Safety outcomes included any reported bleeding events, including intracranial hemorrhage and gastrointestinal bleeding. Only studies published in English were included for review of the full text. Studies presented only in conference abstracts without an associated publication were excluded, as this data is not peer reviewed and there is often limited information available on the details of the study and patient characteristics. A PRISMA flow diagram was created based on the search results and study selection.

2.3. Data Extraction and Quality Assessment. Relevant information was extracted by two independent reviewers using the Doctor Evidence software platform version 2.0 [16]. The following information was collected: study design, study location, publication year, number of patients in each arm, intervention, comorbidities, outcomes of interest, and study inclusion/exclusion criteria. When available, the definitions and descriptions of stroke and outcomes provided by the authors were also captured. Any discrepancies in data extraction were resolved by discussion. All terms (characteristics and outcomes) were collected as reported by study authors and synonyms were "bound" before analysis using the $\mathrm{DOC}^{\mathrm{rm}}$ Ontology System. Detailed methods are described elsewhere [16].

Quality assessment of the included studies was performed by two independent reviewers using the Cochrane Collaboration tool for assessing the risk of bias for randomized trials and the Newcastle-Ottawa Scale for cohort studies $[18,19]$.

2.4. Statistical Analysis. Pairwise meta-analysis for outcomes were performed using the inverse-variance weighted random effects model based on the DerSimonian and Laird 1986 method to estimate the risk ratio (RR) and 95\% confidence interval (CI) [20]. A random effects model took into account both within-study and between-study variability. Heterogeneity was assessed using the $I$-squared $\left(I^{2}\right)$ statistic, which describes the percentage of variation across the studies that is due to heterogeneity rather than chance [21]. Percentages of approximately $25 \%, 50 \%$, and $75 \%$ were considered to have low, moderate, or high heterogeneity, respectively. Patient and 
study characteristics were visually assessed for any potential heterogeneity across studies that might have affected pooled effect estimates. Publication bias could not be assessed due to the limited number of studies available for all outcomes. Multiple analyses were also conducted to consider varying definitions for the composite MACCE outcome in the event that studies reported more than one composite outcome that could qualify as MACCE. If a study reported multiple composite vascular outcomes, the most inclusive composite outcome was selected for the main analysis [22]. All analyses were performed on DOC ${ }^{\mathrm{rm}}$ Data, using R (metaphor package [v.2.0.0]) [23].

\section{Results}

3.1. Literature Search. Of the 2,790 records identified in our search, 2,742 were excluded through title and abstract screening. One additional study was identified manually after the initial search [24]. Among the 48 full texts reviewed, six studies met eligibility criteria (Figure 1). One paper [21] reported on diabetic patients for the same retrospective cohort [22] and was therefore excluded, resulting in a total of five studies included in the meta-analysis.
3.2. Quality Assessment. The quality assessment for the included studies is presented in Supplemental Table II. The risk of bias was rated as low for all seven domains for the RCT. All of the observational studies were rated as of high quality, with Newcastle Ottawa Scale scores of eight or nine. The $I^{2}$ statistic for each outcome is shown in Figures 2 and 3.

3.3. Study and Patient Characteristics. One RCT and four retrospective cohort studies met the PICO criteria for inclusion. Study and patient characteristics of the studies are shown in Table 2. All retrospective cohort studies were conducted in a single country (Denmark, Greece, and Taiwan), whereas the RCT was conducted across 16 countries. All studies were published in peer-reviewed journals.

A total of 29,357 adult patients who had recent ischemic stroke received either clopidogrel $(n=14,293)$ or aspirin $(n=15,064)$ for secondary prevention. The proportion of males ranged from $48 \%$ to $73 \%$. The mean age ranged from 64.5 years to 77.6 years. The length of study follow-up ranged from one year to five years. Comorbidities such as diabetes, hypertension, hyperlipidemia, coronary artery disease, myocardial infarction, congestive heart failure, and peripheral artery disease were prevalent at baseline (Table 2). There was

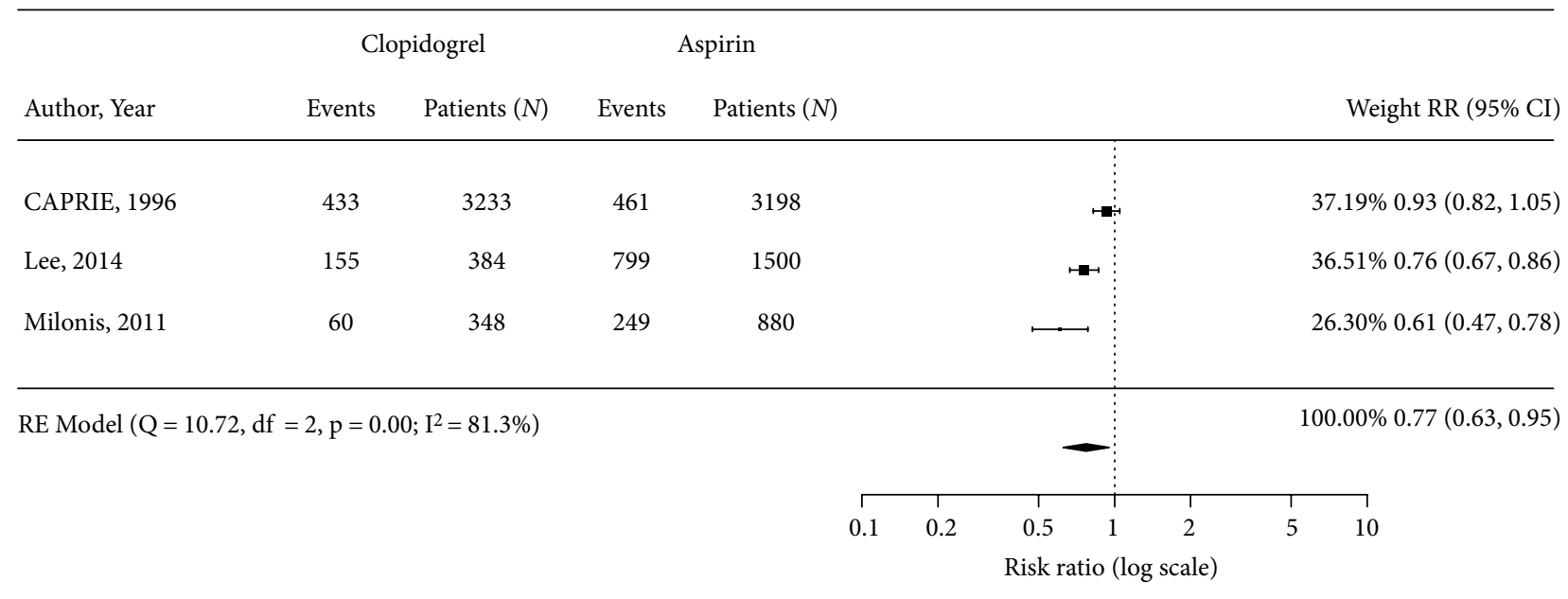

(a)

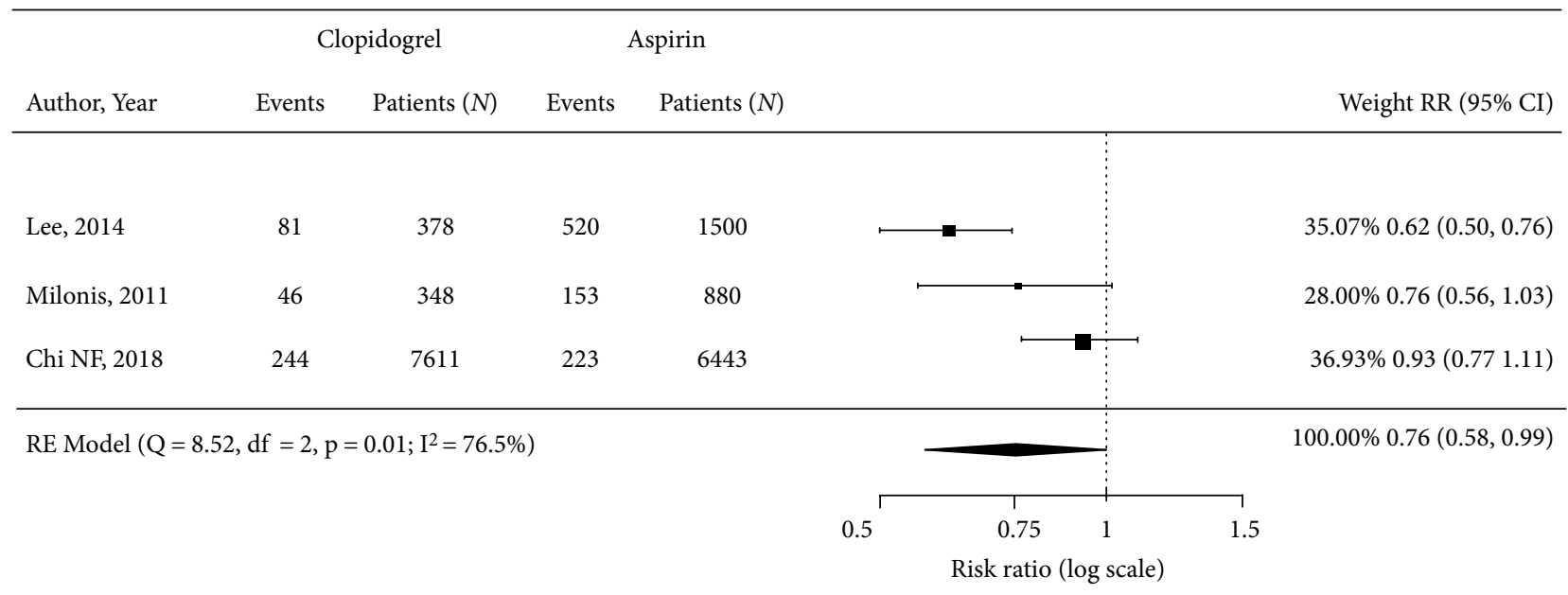

(b)

FIgure 2: Continued. 


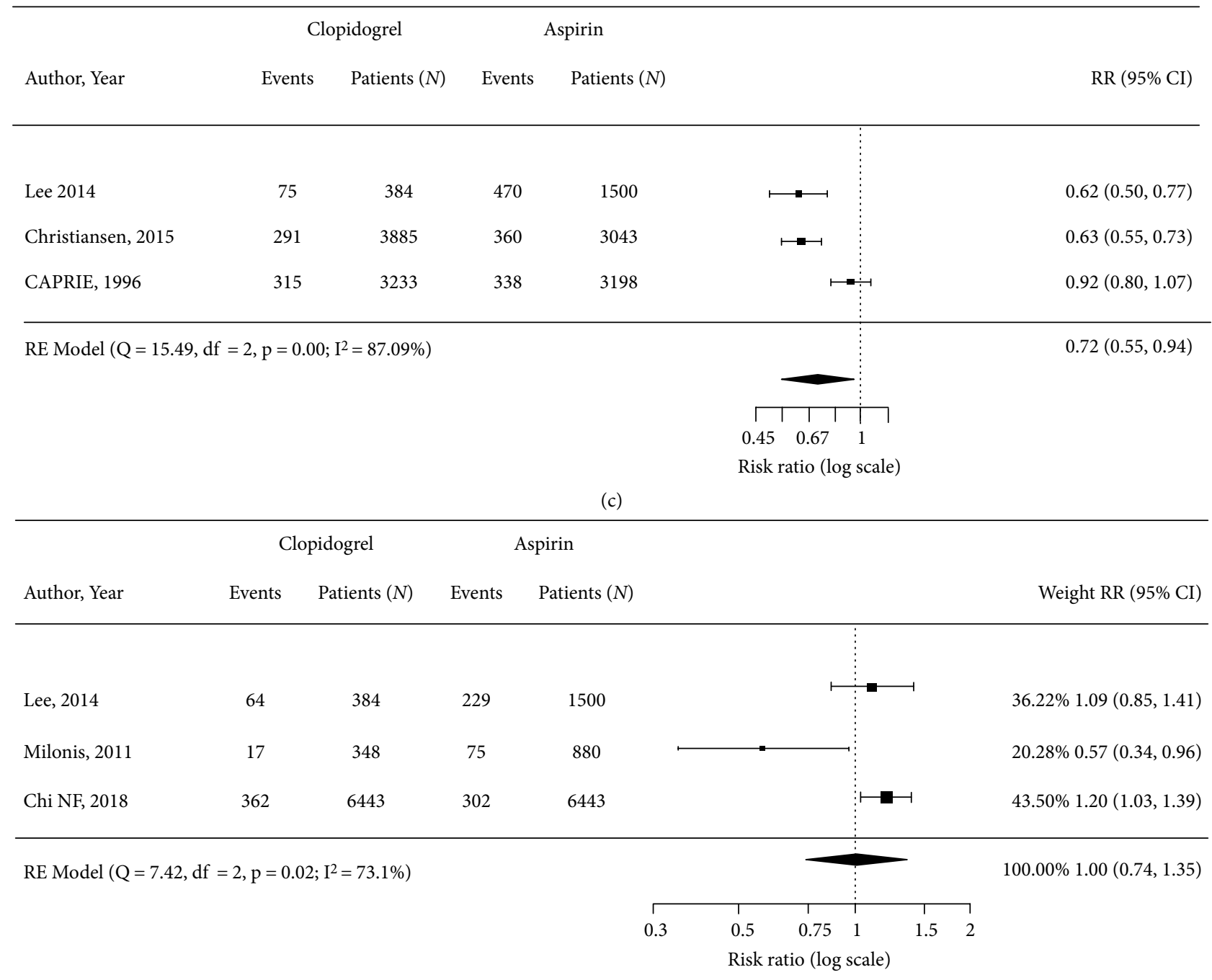

(d)

FIGURE 2: Forest plots showing pooled risk ratio of (a) MACCE, (b) any ischemic or hemorrhagic stroke, (c) recurrent ischemic stroke, and (d) all-cause mortality.

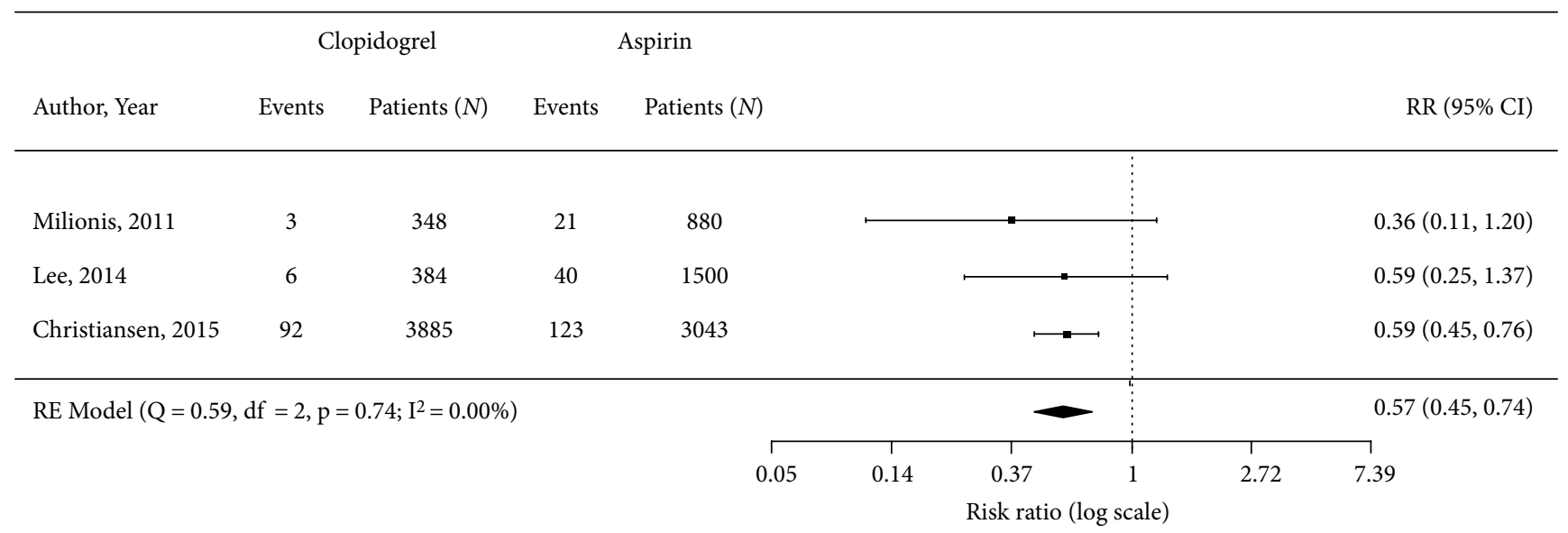

FIGURE 3: Forest plot showing pooled risk ratio for bleeding events. 
no observed trend in different patient characteristics between two treatment groups upon inspection. Two studies did not enroll patients with a history of atrial fibrillation $[22,25]$ and three studies did not enroll patients who had received anticoagulation therapy [24-26]. The average daily dose of clopidogrel was similar across the studies that reported the average daily dose ( $75 \mathrm{mg} /$ day $)$, whereas the average daily dosage of aspirin varied from $102 \mathrm{mg} /$ day to $325 \mathrm{mg} /$ day. The reported outcome definitions varied across studies, most notably for MACCE and bleeding events (Supplemental Table III).

3.4. Comparative Efficacy and Effectiveness. Results of the pairwise meta-analysis showed a statistically significantly lower risk of MACCE among patients who received clopidogrel compared to those who received aspirin (RR 0.77 [95\% CI, $0.63,0.95]$; Figure 2(a)). The risks of stroke of any type, ischemic or hemorrhagic, $(0.76$ [0.58, 0.99]; Figure 2(b)), and recurrent ischemic stroke $(0.72[0.55,0.94]$; Figure $2(c))$ were statistically significantly lower with clopidogrel therapy. There was no difference found for the rate of all-cause mortality (Figure 2(d)).

Sensitivity analysis using a more restrictive definition for MACCE reported in Lee et al. 2014 (i.e., ischemic or hemorrhagic stroke or MI) showed a similar result; patients receiving clopidogrel experienced a lower rate of MACCE (0.72 [0.47, $0.78]$ ) compared to aspirin (Supplemental Table IV and Supplemental Figure I).

3.5. Safety. Bleeding events were reported in three studies (Supplemental Table V). Statistically significant reduction in risk of bleeding events was shown for clopidogrel (0.57 [0.45, 0.74]) compared to aspirin (Figure 3).

\section{Discussion}

The results of our review suggest clinical benefit for single antiplatelet therapy with clopidogrel over aspirin in recent ischemic stroke patients. Pooled relative risk estimates for major composite cardiovascular and cerebrovascular events, recurrence of ischemic stroke, or any ischemic or hemorrhagic stroke were all significantly lower for clopidogrel monotherapy compared to aspirin. Risk of bleeding events were also significantly lower with clopidogrel therapy.

Although broad searches were conducted to identify and include all available literature and sensitivity analyses were run to test the robustness of our findings, there are some limitations to be considered when interpreting these results. Studies often reported composite outcomes as their primary outcome because a smaller sample size is required to adequately power a composite outcome as compared to individual outcomes. Definitions of MACCE and recurrent stroke that most closely resembled the definitions reported in other studies were used, but data collection was limited to the published study-level results. The between-study heterogeneity found in our analyses remain unexplained due to the nature of observational studies. Thus, the pooled preventative effects of clopidogrel over aspirin shown for MACCE and recurrent stroke may underestimate the true preventative effects of clopidogrel.

Bleeding event data was only available from retrospective cohort studies. Any reported bleeding events including both composite bleeding events and specific bleeding events were combined (e.g., intracranial hemorrhage and gastrointestinal bleeding). These bleeding events were captured from insurance databases or national registries, and it is reasonable to assume that these events were severe enough to require medical attention (e.g., office visit or hospitalization). However, due to the nature of the claim-based database and national registries, we were unable to compare bleeding events by severity. In one study [22], the authors noted that clopidogrel was prescribed only for those with pre-existing gastrointestinal ulcers or bleeding issues or for those who have already failed on aspirin. This is important to note as the results may not reflect the true rate of bleeding events associated with clopidogrel use.

Selecting optimal therapy for secondary stroke prevention requires careful attention, as these patients often present with comorbidities and other risk factors which may influence prescription and treatment effectiveness. The boxed label warning for clopidogrel cautions against use of clopidogrel in patients with impaired platelet reactivity due to known genetic polymorphisms of CYP2C19 [28]. The majority of studies in this review included data from before 2010 and genotype testing or platelet monitoring via platelet function tests may not have been performed, as routine testing is still not included in any current guideline recommendations. The included observational studies used claim-based or registry data, and in such real-world settings, the selection of antiplatelets was based on physicians' preference and receipt of clopidogrel often depended on insurance or drug formularies and requirements by country. However, this information was not reported in the studies. Due to the nature of the retrospective cohort studies included in this analysis, clinicians should be aware that unknown and therefore unmeasured confounders might have affected our effect estimates differentially.

Aspirin remains the recommended antiplatelet therapy for patients with ischemic stroke in current guidelines [8]. Published trial data suggests clopidogrel as single antiplatelet therapy is safe and effective for secondary prevention compared to aspirin and the combination of aspirin/dipyridamole $[27,29]$. However, the strength of the evidence in support of clopidogrel over other antiplatelet agents is limited by the few numbers of studies that make direct comparison to clopidogrel as single antiplatelet therapy, and more recent data is based on the combination of clopidogrel and aspirin in mixed populations with ischemic stroke or TIA. In the absence of further clinical trials, indirect evidence obtained through further meta-analysis and data from prospective patient registries may provide valuable insights on the efficacy and safety of clopidogrel relative to aspirin for secondary prevention patients with ischemic stroke.

To our knowledge, this is the first systematic review and meta-analysis conducted in patients with recent ischemic stroke specifically, as most of the current evidence is based on stroke and TIA populations. We included all published clinical trials and observational studies that made direct comparison of clopidogrel and aspirin monotherapy for secondary 


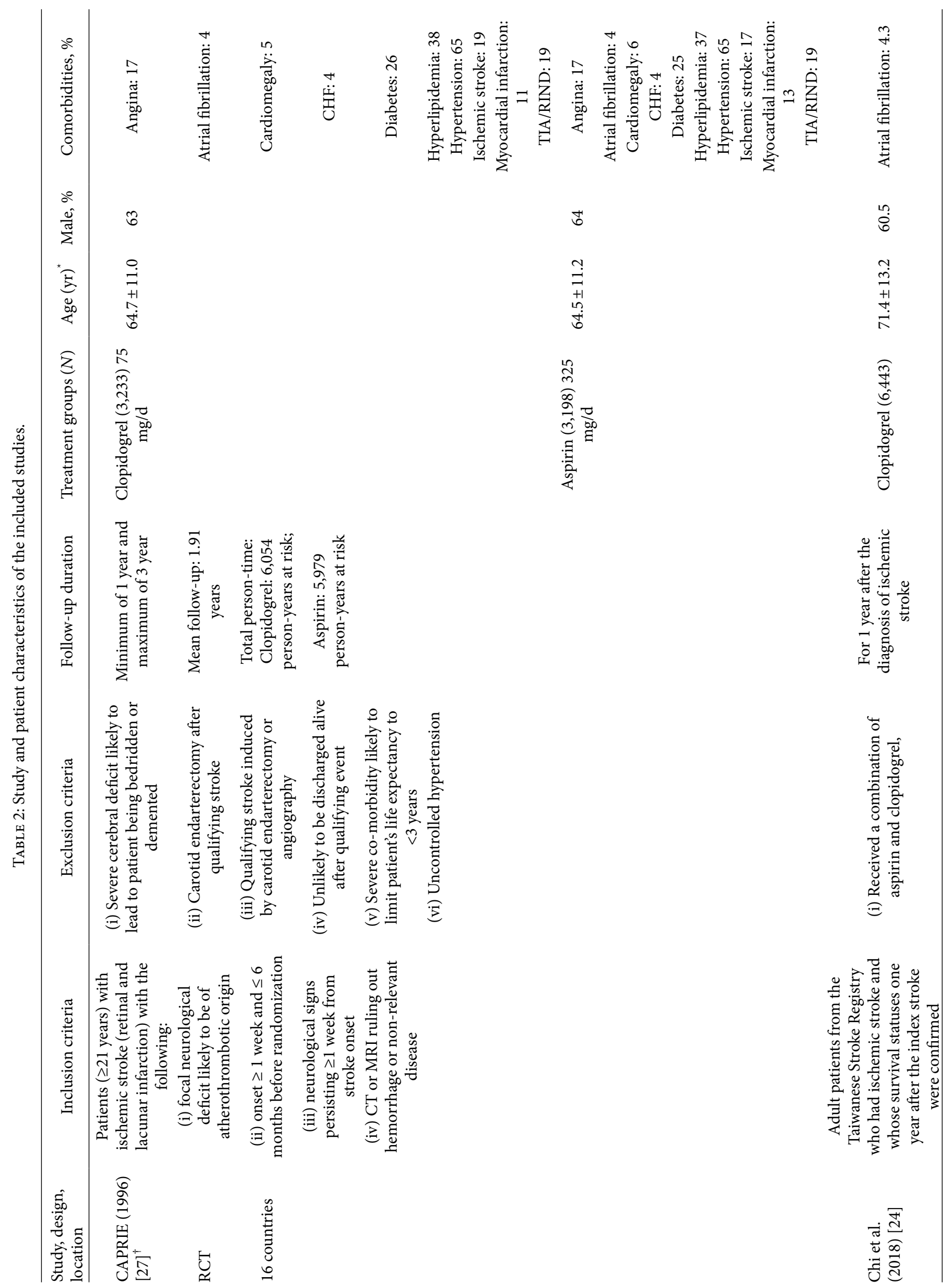




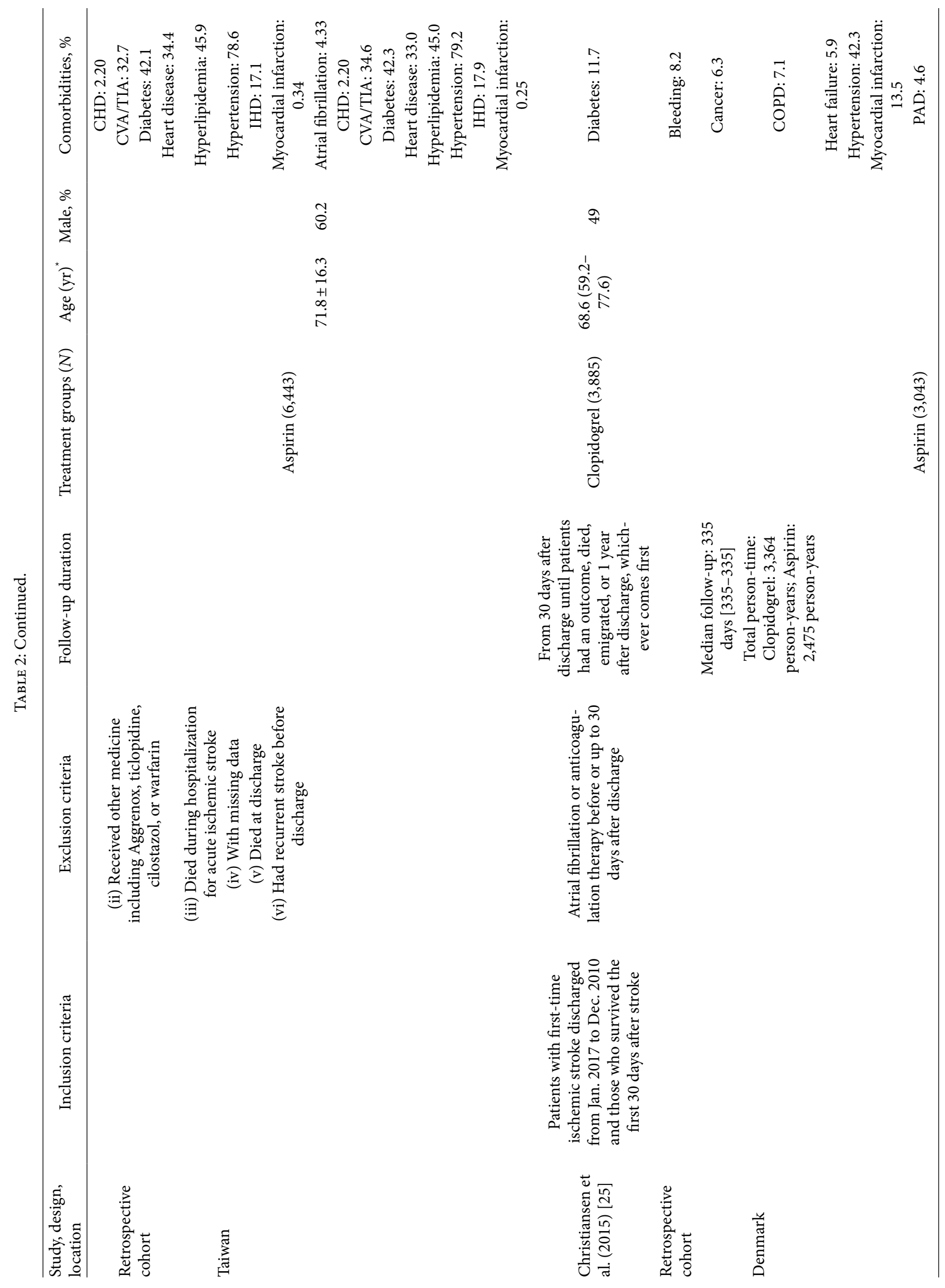




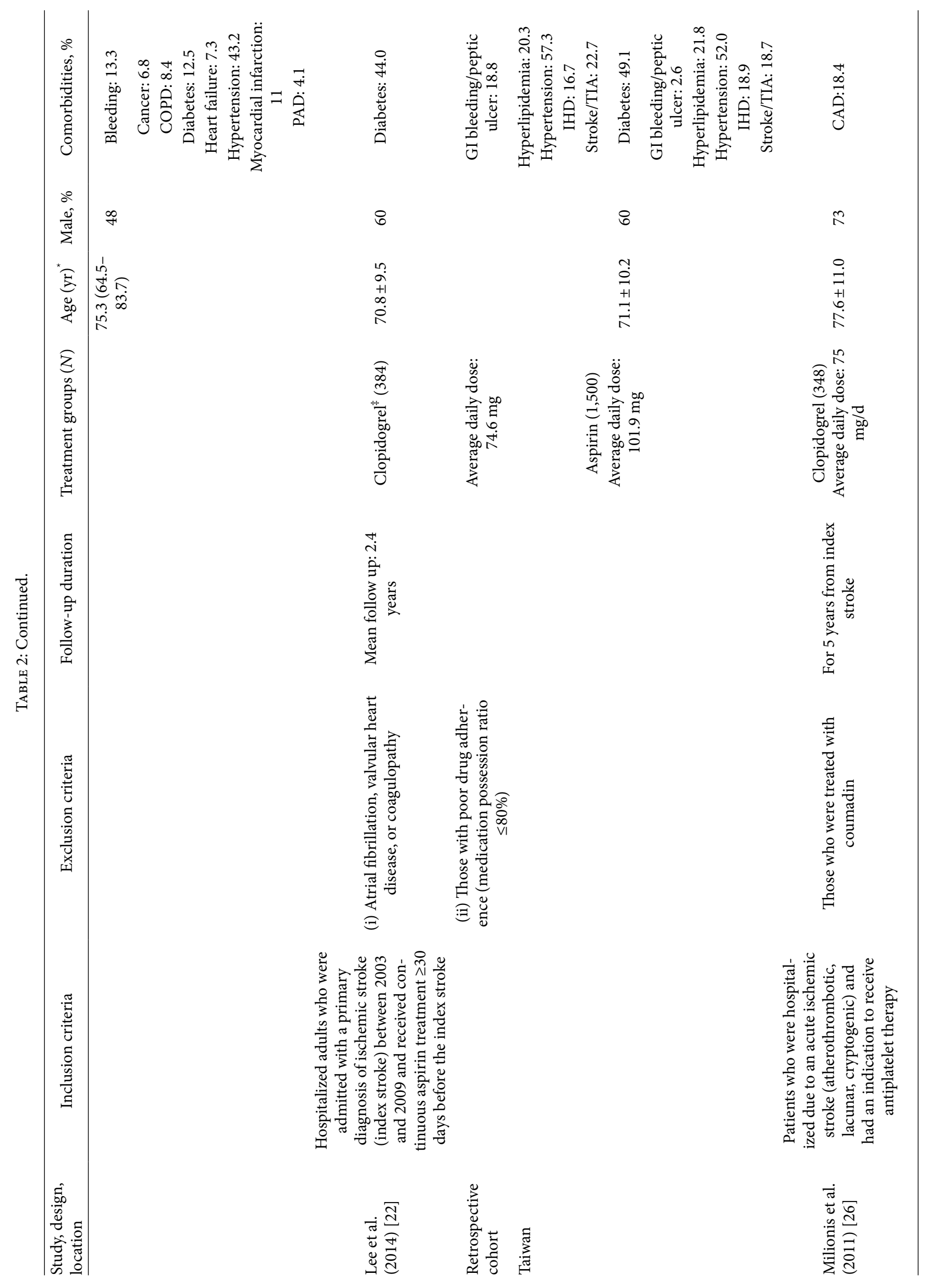




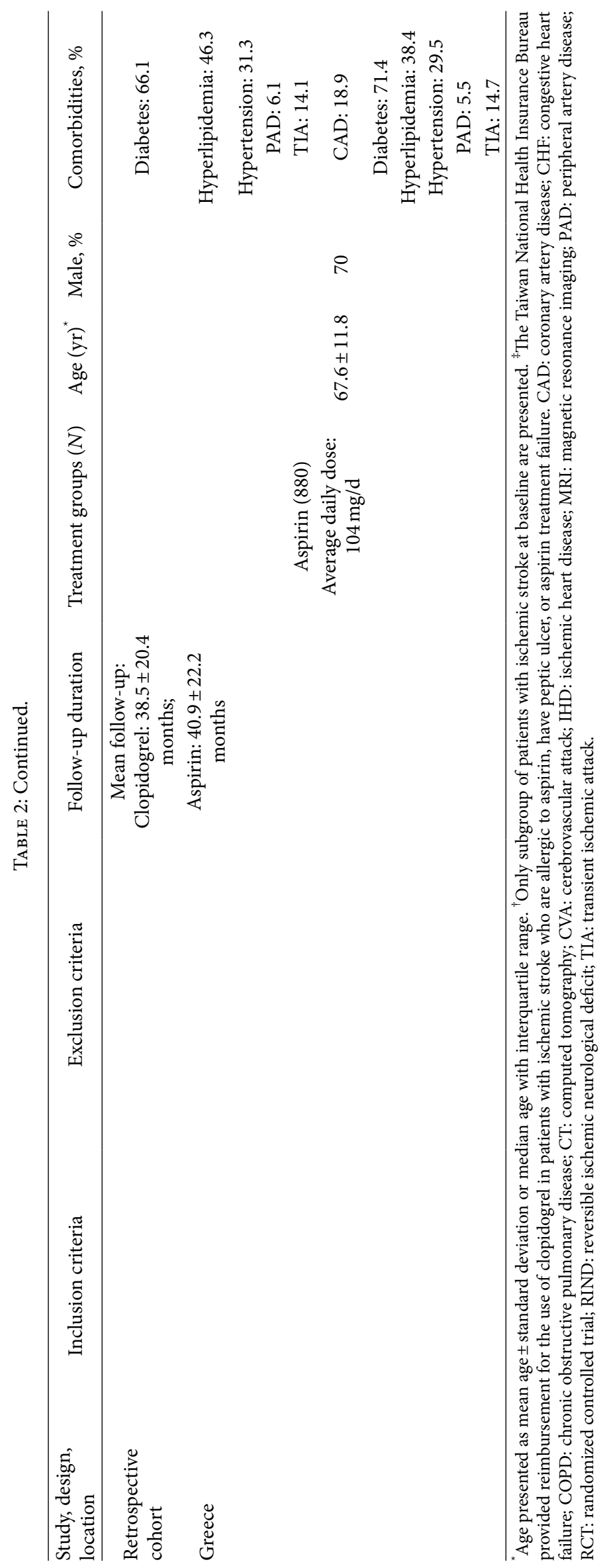


prevention in patients with ischemic stroke. Data from observational studies were included to explore the benefits and harms for this population in the real-world setting. Because there is limited data on the relative efficacy and safety of clopidogrel compared to aspirin alone, these findings can add valuable information to help clinicians and policymakers in selection of antiplatelet therapy for secondary prevention following recent ischemic stroke.

\section{Conclusions}

This systematic review and meta-analysis found that clopidogrel monotherapy was associated with significantly lower risks of MACCE, recurrent stroke, and bleeding events compared to aspirin in patients with ischemic stroke. The results of the analysis support clinical benefit for single antiplatelet therapy with clopidogrel over aspirin for secondary prevention in patients with recent ischemic stroke. There were few studies included in this review and data were based largely on retrospective observational data. More longitudinal data and high-quality studies are warranted to verify the findings of this systematic literature review and meta-analysis.

\section{Conflicts of Interest}

Maurizio Paciaroni received honoraria as a member of the speaker bureau of Aspen, Sanofi-Aventis, Boehringer Ingelheim, Bayer, Bristol Meyer Squibb, Daiiki Sankyo and Pfizer. Birsen Ince has received honoraria from Pfizer and Sanofi-Aventis within the past 36 months, outside the submitted work. Kursad Kutluk has received honoraria from Boehringer Ingelheim, Pfizer and Sanofi-Aventis within the past 36 months, outside the submitted work. Dara Paek, Jung Min Han, and Michael del Aguila report employment by Doctor Evidence at the time in which this research was conducted, who was contracted by Sanofi to perform the analysis. Shalini Girotra reports employment by Sanofi. All other authors have nothing to disclose.

\section{Authors' Contributions}

All authors met ICMJE guidelines for authorship and provided analysis interpretation, critical review, revision, and final approval of the article. M. Paciaroni, D. Paek, M. del Aguila, and S. Girotra aided in the concept/design of the study. D. Paek, J. M. Han, and M. del Aguila also aided in data collection and analysis. The authors thank Toby Sayre of Doctor Evidence for medical writing and support and Angelica Stamegna for general publication management.

\section{Funding}

This study was funded by Sanofi.

\section{Supplementary Materials}

The supplementary PDF includes summaries of the search strings, in addition to further information on methodology and results. (Supplementary Materials)

\section{References}

[1] V. L. Feigin, G. A. Roth, M. Naghavi et al., "Global burden of stroke and risk factors in 188 countries, during 1990-2013: a systematic analysis for the Global Burden of Disease Study 2013," The Lancet Neurology, vol. 15, pp. 913-924, 2016.

[2] E. J. Benjamin, M. J. Blaha, S. E. Chiuve et al., "Heart disease and stroke statistics-2017 update: a report from the American Heart Association," Circulation, vol. 135, pp. e146-e603, 2017.

[3] W. Feng, R. M. Hendry, and R. J. Adams, "Risk of recurrent stroke, myocardial infarction, or death in hospitalized stroke patients," Neurology, vol. 74, no. 7, pp. 588-593, 2010.

[4] N. M. Engel-Nitz, S. D. Sander, C. Harley, G. G. Rey, and H. Shah, "Costs and outcomes of noncardioembolic ischemic stroke in a managed care population," Vascular Health and Risk Management, vol. 6, pp. 905-913, 2010.

[5] J. Burn, M. Dennis, J. Bamford, P. Sandercock, D. Wade, and C. Warlow, "Long-term risk of recurrent stroke after a first-ever stroke. The Oxfordshire Community Stroke Project," Stroke, vol. 25, pp. 333-337, 1994.

[6] G. P. Samsa, J. Bian, J. Lipscomb, and D. B. Matchar, "Epidemiology of recurrent cerebral infarction: a Medicare claims-based comparison of first and recurrent strokes on 2-year survival and cost," Stroke, vol. 30, no. 2, pp. 338-349, 1999.

[7] W. N. Kernan, B. Ovbiagele, H. R. Black et al., "Guidelines for the prevention of stroke in patients with stroke and transient ischemic attack: a guideline for healthcare professionals from the American Heart Association/American Stroke Association," Stroke, vol. 45, no. 7, pp. 2160-2236, 2014.

[8] W. J. Powers, A. A. Rabinstein, T. Ackerson et al., "2018 guidelines for the early management of patients with acute ischemic stroke: a guideline for healthcare professionals from the American Heart Association/American Stroke Association," Stroke, vol. 49, no. 3, 2018.

[9] R. G. Hart, L. A. Pearce, and M. I. Aguilar, "Meta-analysis: antithrombotic therapy to prevent stroke in patients who have nonvalvular atrial fibrillation," Annals of Internal Medicine, vol. 146, no. 12, pp. 857-867, 2007.

[10] P. Gorelick, O. Sechenova, and C. H. Hennekens, "Evolving perspectives on clopidogrel in the treatment of ischemic stroke," Journal of Cardiovascular Pharmacology and Therapeutics, vol. 11, no. 4, pp. 245-248, 2006.

[11] A. Algra and J. van Gijn, "Is clopidogrel superior to aspirin in secondary prevention of vascular disease?" Trials, vol. 1, no. 3, p. 143, 2000.

[12] A. Algra and J. Van Gijn, "Aspirin at any dose above $30 \mathrm{mg}$ offers only modest protection after cerebral ischaemia," Journal of Neurology, Neurosurgery \& Psychiatry, vol. 60, no. 2, pp. 197-199, 1996.

[13] A. Algra and J. Van Gijn, "Cumulative meta-analysis of aspirin efficacy after cerebral ischaemia of arterial origin," Journal of Neurology, Neurosurgery, and Psychiatry, vol. 66, no. 2, p. 255, 1999. 
[14] Y. Wang, Y. Pan, X. Zhao et al., "Clopidogrel with aspirin in acute minor stroke or transient ischemic attack (CHANCE) trial: one-year outcomes," Circulation, vol. 132, no. 1, pp. 40-46, 2015.

[15] Y. Wang, Y. Wang, X. Zhao et al., "Clopidogrel with aspirin in acute minor stroke or transient ischemic attack," New England Journal of Medicine, vol. 369, no. 1, pp. 11-19, 2013.

[16] P. J. Mazzone, G. A. Silvestri, S. Patel et al., "Screening for lung cancer: CHEST guideline and expert panel report," Chest, vol. 153, no. 4, pp. 954-985, 2018.

[17] L. Shamseer, D. Moher, M. Clarke et al., "Preferred reporting items for systematic review and meta-analysis protocols (PRISMA-P) 2015: elaboration and explanation," BMJ, vol. 349, p. g7647, 2015.

[18] J. P. T. Higgins, D. G. Altman, P. C. Gøtzsche et al., "The Cochrane Collaboration's tool for assessing risk of bias in randomised trials," BMJ, vol. 343, 2011.

[19] G. Wells, B. Shea, D. O'connell et al., "The Newcastle-Ottawa Scale (NOS) for assessing the quality of nonrandomized studies in meta-analysis," 2011, http://www.ohri.ca/programs/clinical_ epidemiology/oxford.asp.

[20] R. DerSimonian and N. Laird, "Meta-analysis in clinical trials," Controlled Clinical Trials, vol. 7, no. 3, pp. 177-188, 1986.

[21] J. P. T. Higgins and S. G. Thompson, "Quantifying heterogeneity in a meta-analysis," Statistics in Medicine, vol. 21, no. 11, pp. 1539-1558, 2002.

[22] M. Lee, Y. L. Wu, J. L. Saver et al., "Is clopidogrel better than aspirin following breakthrough strokes while on aspirin? a retrospective cohort study," BMJ Open, vol. 4, no. 12, p. e006672, 2014.

[23] W. Viechtbauer, "Conducting meta-analyses in $\mathrm{R}$ with the meta for package," Journal of Statistical Software, vol. 36, no. 3, pp. 1-48, 2010.

[24] N.-F. Chi, C.-P. Wen, C.-H. Liu et al., "Comparison between aspirin and clopidogrel in secondary stroke prevention based on real-world data," Journal of the American Heart Association, vol. 7, no. 19, 2018.

[25] C. B. Christiansen, J. Pallisgaard, T. A. Gerds et al., "Comparison of antiplatelet regimens in secondary stroke prevention: a nationwide cohort study," BMC Neurolology, vol. 15, no. 1, p. 225, 2015.

[26] H. J. Milionis, G. Gerotziafas, M. S. Kostapanos et al., "Clopidogrel vs. aspirin treatment on admission improves 5-year survival after a first-ever acute ischemic stroke. Data from the Athens Stroke Outcome Project," Archives of Medical Research, vol. 42, no. 6, pp. 443-450, 2011.

[27] CAPRIE Steering Committee, "A randomised, blinded, trial of clopidogrel versus aspirin in patients at risk of ischaemic events (CAPRIE)," Lancet, vol. 348, no. 9038, pp. 1329-1339, 1996.

[28] FDA Drug Safety Communication, "Reduced effectiveness of Plavix (clopidogrel) in patients who are poor metabolizers of the drug," 2010, http://www.fda.gov/Drugs/DrugSafety/ PostmarketDrugSafetyInformationforPatientsandProviders/ ucm203888.htm.

[29] R. L. Sacco, H. C. Diener, S. Yusuf et al., "Aspirin and extendedrelease dipyridamole versus clopidogrel for recurrent stroke," New England Journal of Medicine, vol. 359, pp. 1238-1251, 2008. 


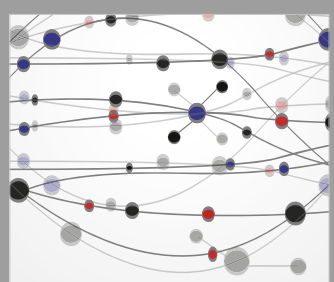

The Scientific World Journal
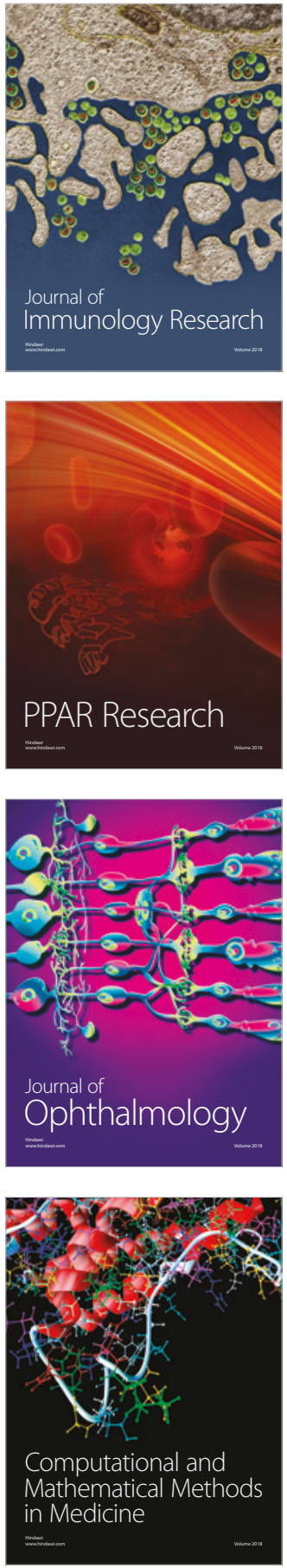

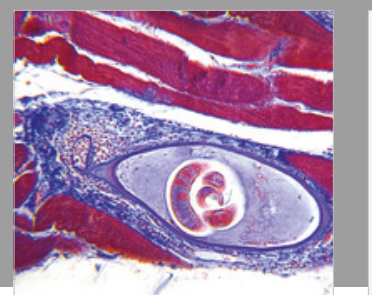

Gastroenterology Research and Practice

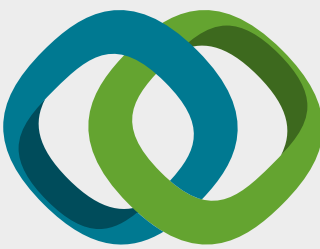

\section{Hindawi}

Submit your manuscripts at

www.hindawi.com
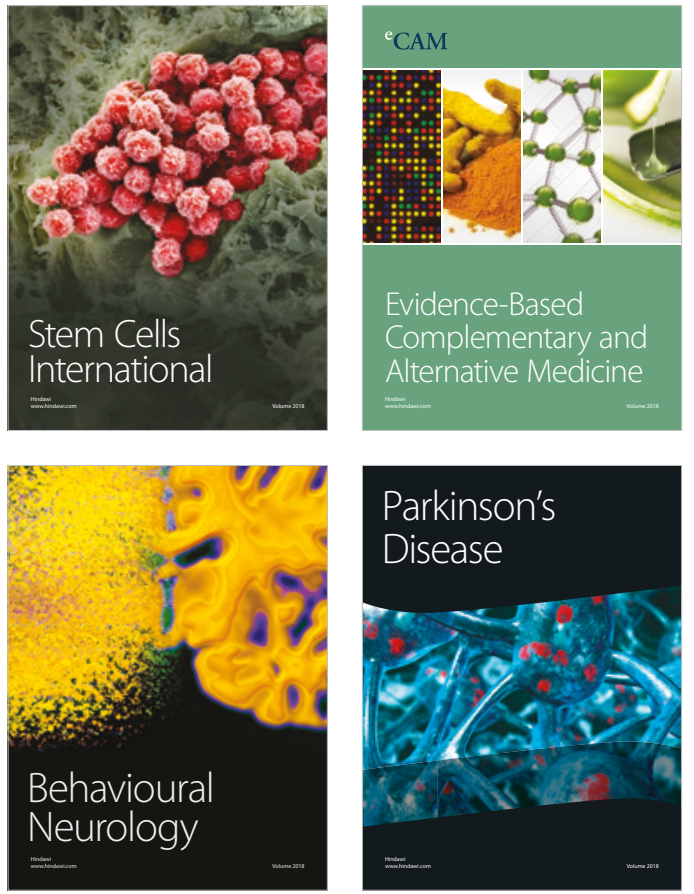

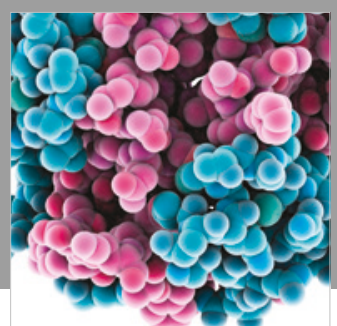

ournal of

Diabetes Research

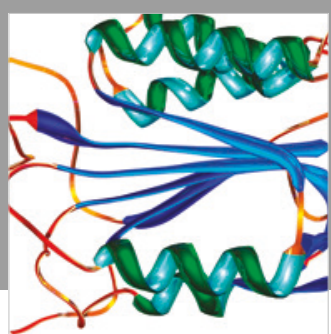

Disease Markers
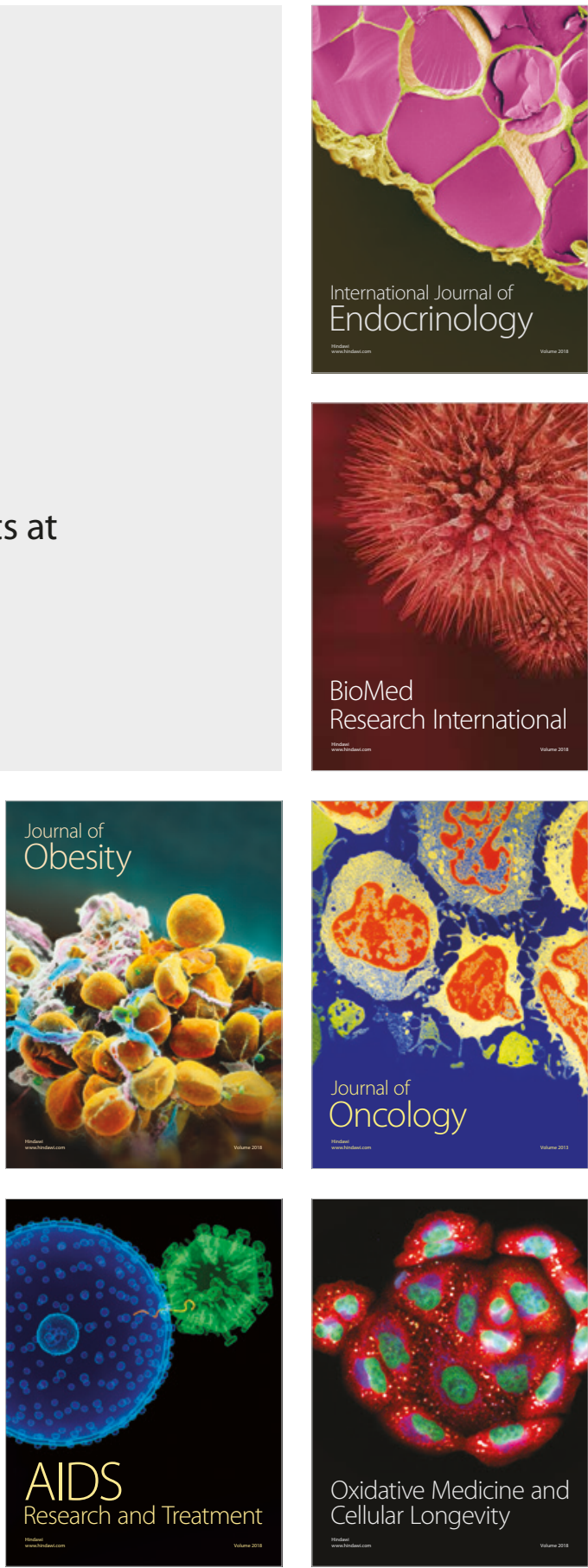